\title{
Research of thermal conditions over high- temperature gas-fired infrared emitters
}

\author{
Anton N. Ermolaev ${ }^{1}$, Sergey A. Khaustov ${ }^{2, *}$ \\ ${ }^{1}$ Tyumen industrial University, 625000 Tyumen, Russia \\ ${ }^{2}$ National Research Tomsk Polytechnic University, 634050 Tomsk, Russia
}

\begin{abstract}
The paper presents the study results of the thermal conditions in the area above high-temperature gas-fired infrared emitter. A number of bench tests and experiments were made on the basis of production facilities to control the distribution of temperatures above emitter in different heating system operating modes. Impact of the thermal characteristics in the area above high-temperature gas-fired infrared emitter on the heating system performance was estimated. Comparison of the bench tests results with existing experimental data has shown a good result convergence for both efficiency and accuracy. The obtained results can be used in the emitter development phase and in the construction phase of modern gasfired radiant heating systems.
\end{abstract}

\section{Introduction}

Recently, there are increasingly used radiant heating systems based on high-temperature gas-fired infrared emitters with the goal of energy resource saving [1, 2]. This heating method has significant benefits in comparison with traditional convection heating systems $[3,4]$. Modern radiant heating systems based on gas-fired infrared heat emitters lead to high energy efficiency when heating large-scale production buildings $[1,5]$.

Despite the fact that contemporary high-temperature gas-fired infrared emitters have high energy efficiency, during their operation for heating large-scale production buildings a number of drawbacks was discovered [5,6]: upper zone was overheated; smut appeared on walling surface resulting in wall subsequent destruction. Listed drawbacks are caused by considerable heat losses with stack gases (24-30\%) and by emitter design (9-18\%). Strauch-Sürken, Michael Wendt. Tierärztliche Praxis suggest that heat-insulating properties of reflector material effects the radiant heating system, and propos a self-brazing aluminum clad steel [7] for producing gas-fired infrared emitter's reflecting surfaces.

We carried out a series of experiments on specially designed experimental setup to obtain temperature fields formed in the area above high-temperature gas-fired infrared emitter's installation. Object of the research were high-temperature gas-fired infrared emitters with thermal capacity of $5,10,15,20,30,40 \mathrm{~kW}$.

\footnotetext{
*Corresponding author: khaustovsa@tpu.ru
} 


\section{Experimental setup}

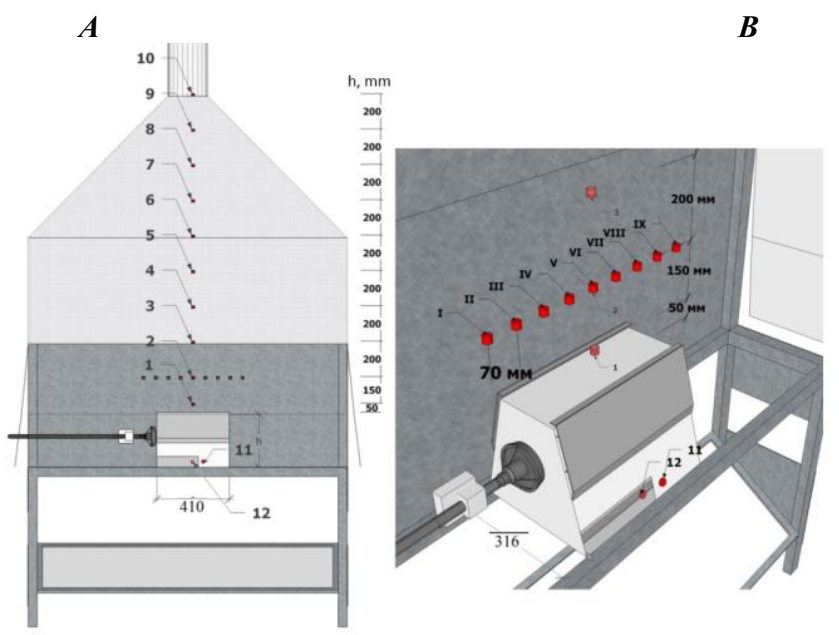

Fig. 1. Longitudinal section (A) and isometric view (B) of experimental setup: numbers from 1 to 10 indicate vertical positions of thermocouples, roman numbers from I to IX indicate horizontal positions of thermocouples, sensors numbered with 11 and 12 are spring-loaded probes Testo-480 for measuring the casing surface temperatures.

A gaseous medium inside the gas-fired infrared heat emitter was carried out on an experimental setup shown in Fig.1. The heating control was implemented with certified instrumentation. Thermoelectric converters THC-0006 were used for measuring the temperature of the gaseous medium and a spring-loaded probe Testo-480, for measuring the casing surface temperatures.

During the experiment, a vertical pitch between the sensors remained unchanged, $\mathrm{x}=$ $100 \mathrm{~mm}$. The horizontal pitch between the sensors was adjusted in proportion to the heat output of the emitter. The heat output at different horizontal pitches is shown in table 1.

Table 1. The heat output at different horizontal pitches.

Heat output
$5 \mathrm{~kW}$
$10 \mathrm{~kW}$
$15 \mathrm{~kW}$
$20 \mathrm{~kW}$
$30 \mathrm{~kW}$
$40 \mathrm{~kW}$

$$
\begin{gathered}
\text { Horizontal pitch } \\
70 \mathrm{~mm} \\
115 \mathrm{~mm} \\
160 \mathrm{~mm} \\
205 \mathrm{~mm} \\
180 \mathrm{~mm} \\
225 \mathrm{~mm}
\end{gathered}
$$

Experimental data from the sensors was transferred to PC by an analog-digital converter for Finite Element Comparison.

Gas-fired infrared heat emitters with heat output of 5, 10, 15, 20, 30, $40 \mathrm{~kW}$ were studied. The gas pressure before the inlet nozzle was $1270 \mathrm{~Pa}$. The air-fuel ratio (AFR) was 9.996. Tests of each high-temperature gas-fired infrared emitter's thermal capacity were carried out for 40 minutes with temperature recording for every 40 seconds after launching. This allowed to study the thermal conditions change above the emitter before and after stationary conditions reaching. All high-temperature gas-fired infrared emitter's tech specs are taken according to nominal data. 


\section{Results of the study}

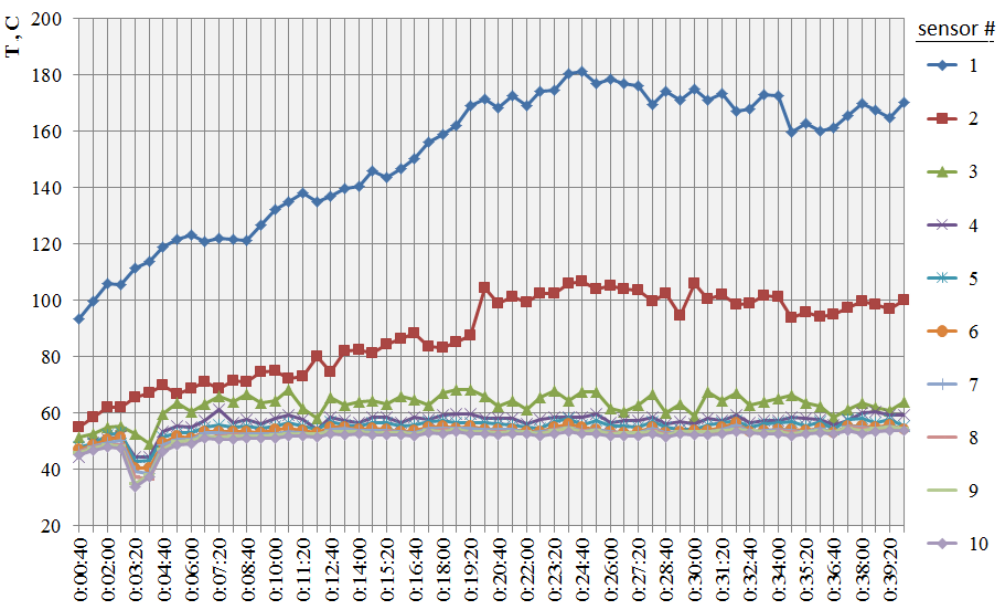

Fig. 2. Temperature distribution in locations of the sensors 1-10 over $5 \mathrm{~kW}$ high-temperature gasfired infrared emitters before and after stationary conditions reaching.

Fig. 2 shows that temperature pulses in time. This pulsation range characterizes turbulent flows appearing above high-temperature gas-fired infrared emitter. The free turbulent flow is caused by gas pressure difference at the exit of region bounded by reflector (excess) and above high-temperature gas-fired infrared emitter (vacuum). These pulsations of temperature in time are peculiar to all thermal capacities. Transition to sustainable mode was reached after 20 minutes of continuous operation under full thermal charge.

It should be noticed that thermal capacity of high-temperature gas-fired infrared emitters is defined by a number of perforated ceramic tiles, unit capacity $(1.5 \mathrm{~kW}$ at an average). The tile surface temperature at an average is $900{ }^{\circ} \mathrm{C}$ and depends on gas pressure in front of the gas-infrared burner nozzle and the ceramic tile composition.

The maximum value of the temperature is recorded at a distance of $50 \mathrm{~mm}$ above the gas-fired infrared heat emitter at the location of the sensor \#1. With the distance from the radiator structure (sensors 1-10) increasing the shape of the curve turns into hyperbola. First $600 \mathrm{~mm}$ temperature falls significantly. The difference between the temperatures of the first $600 \mathrm{~mm}$ and at higher distance increases with heat output. The temperature above gas-fired infrared heat emitter $5 \mathrm{~kW}$ is reduced by three times, while for $40 \mathrm{~kW}$, barely 1.5 times.

The temperature distribution along the gas-fired infrared heat emitter (sensors I-IX) is uneven. If heat output increases, the spread in temperature values reduces. The results of temperature measurement on outer (sensor No11) and inner (sensor No12) sides of the reflector surface are shown in table 2.

Table 2. The results of gas-fired infrared emitter's casing temperature measurement.

\begin{tabular}{|c|c|c|}
\hline $\begin{array}{c}\text { High-temperature gas-fired } \\
\text { infrared emitter thermal } \\
\text { capacity, } \mathrm{kW}\end{array}$ & $\begin{array}{c}\text { Casing temperature on outer } \\
\text { side in location of the sensor } \\
\text { No11, }{ }^{\circ} \mathrm{C}\end{array}$ & $\begin{array}{c}\text { Casing temperature on inner } \\
\text { side in location of the sensor } \\
\text { No12, }{ }^{\circ} \mathrm{C}\end{array}$ \\
\hline 5 & 148.08 & 202.81 \\
\hline 10 & 151.04 & 206.86 \\
\hline 15 & 144.93 & 205.06 \\
\hline 20 & 140.59 & 204.85 \\
\hline 30 & 130.80 & 125.18 \\
\hline 40 & 139.15 & 142.40 \\
\hline
\end{tabular}




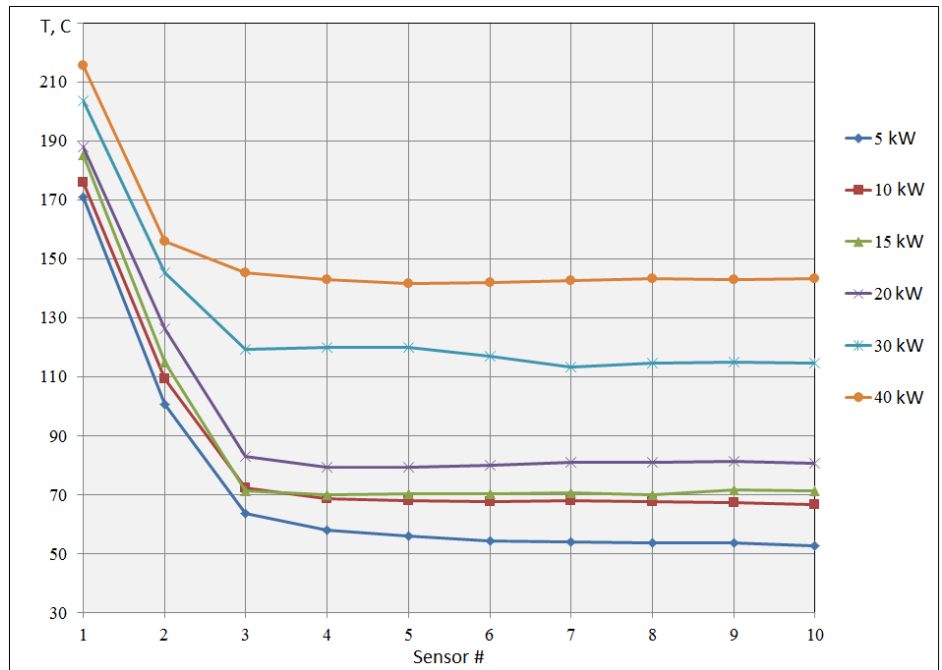

a) vertical sensors $1-10$

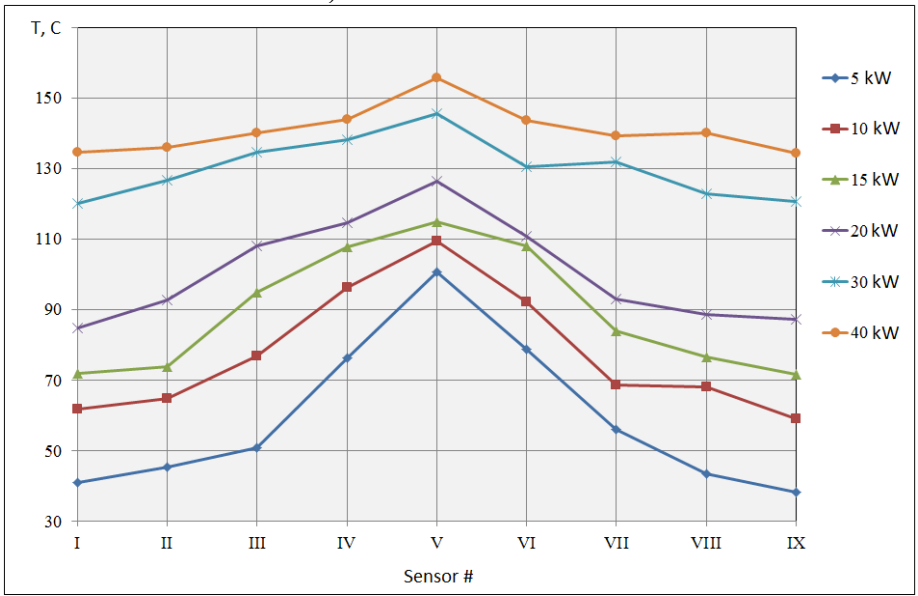

b) horizontal sensors I-IX

Fig. 2. Experimental temperature distribution above 5-40 kW high-temperature gas-fired infrared emitters.

Analysis of the obtained data indicates a feature of high-temperature gas-fired infrared heat emitter. It produces significant thermal radiation in the upper zone of the experimental setup by the heated surfaces with simultaneous flow of convective heat and mass transfer processes.

\section{Conclusion}

According to the obtained results, it can be concluded: reflector temperature on outer and inner sides does not significantly change provided that reflector is made of the same material; temperature change more than $50{ }^{\circ} \mathrm{C}$ inside of $30-40 \mathrm{~kW}$ high-temperature gasfired infrared emitters caused by the use of alternative reflector material. Structural design of 5-20 kW high-temperature gas-fired infrared emitters assumes the use of self-brazing aluminum clad steel with trademark feran ${ }^{\circledR}$, and for 30-40 kW high-temperature gas-fired infrared emitters - polished stainless steel $12 \mathrm{Cr} 18 \mathrm{Ni} 10 \mathrm{Ti}$. Thus, the use of feran ${ }^{\circledR}$ 
increases thermal-insulating properties of the reflector, increasing the heat flow intensity into working area.

A high thermal conductivity of the feran ${ }^{\circledR}$ forming the reflector of gas-fired infrared emitter causes an intensive heat exchange of combustion medium with the outer air. Thus, the temperature distribution at the measuring points over gas-fired infrared emitter becomes uneven in height and time. The thermal conditions above the high-temperature gas-fired infrared emitters depends on its thermal power. The higher thermal output of the burner causes the higher temperatures at each sensor installed. This dependence is explained by the fact that as the thermal power of the gas-fired infrared emitter increases, the proportion of combustion products also increases with respect to the air volume in the experimental setup. As a result, this reduces the intensity of dilution of the combustion products and increases the temperature at each point.

\section{References}

1. Y.M. Luo, L. Chevalier, F. Utheza, X. Nicolas, International Polymer Processing. Journal of the Polymer Processing Society, 30 (5), 554 (2015)

2. M. Ostafin, M. Balkenhol, J. Erlemeyer, M. Köhler, Materialwiss. Werkstofftech. 41, $11(2010)$

3. D. Patino, B. Crespo, J. Porteiro, J.L. Miguez, Appl. Therm. Eng. 100, 849 (2016)

4. S.A. Khaustov, A.V. Kazakov, G.A. Cherkashina, L.A. Sobinova, EPJ Web Conf. 110, 01028 (2016)

5. S. Dolgov, E. Savchenko, S. Khaustov, R. Tabakaev, A. Zavorin, EPJ Web Conf. 110, 01074 (2016)

6. K.A. Al-attab, J.C. Ho, Z.A. Zainal, Exp. Therm. Fluid Sci. 62, 1 (2015)

7. L. Strauch-Sürken, M. Wendt, Tierärztliche Praxis. Ausgabe G, Grosstiere/Nutztiere, 43, 4 (2015) 